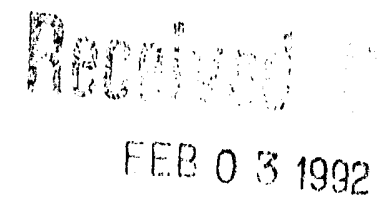

\title{
PIONS IN AND OUT OF EQUILIBRIUM
}

\author{
Sean Gavin \\ Physics Department \\ Brookhaven National Laboratory \\ Upton, New York 11973
}

\begin{abstract}
Nonequilibrium phenomena in pion $p_{T}$ spectra may signal the approach to equilibrium.
\end{abstract}

Presented at Quark Matter 1991

Gatlinburg, TN

11-15 November 1991

(To be published as a special issue of Nuclear Physics A)

This manuscript has been authored under contract number DE-AC02-76CH00016 with the U.S. Department of Energy. Accordingly, the U.S. Government retains a non-exclusive, royalty-free license to publish or reproduce the published form of this contribution, or allow others to do so, for U.S. Government purposes. 


\title{
Pions In and Out of Equilibrium
}

\section{Sean Gavin}

Department of Physics, Brookhaven National Laboratory, Upton, NY 11973, USA

\begin{abstract}
Nonequilibrium phenomena in pion $p_{T}$ spectra may signal the approach to equilibrium.
\end{abstract}

\section{INTRODUCTION}

Can final state scattering wrestle the secondaries in nucleus-rucleus collisions into a fluid state near local thermal equilibrium? What do the pion $p_{T}$ spectra measured in $p p$, $p A$ and SPS light ion experiments already tell us about the approach to equilibrium? To begin to address these questions, we must face the nonequilibrium nature of hadronic evolution in the late stages of these collisions. I will outline efforts to apply transport theory to the nonequilibrium pion fluid at midrapidity focusing on two phenomena: partial tizermalization [1] and pion conservation [2].

Ruuskanen and I [2] have discussed consequences of these nonequilibriurn phenomena in the low $p_{T}$ pion spectra at midrapidity from $\mathrm{O}+\mathrm{Au}$ and $\mathrm{S}+\mathrm{S}$ at $\sqrt{s}=20 \mathrm{GeV}$. Here, new results will be presented comparing high multiplicity to average (minimum bias) $p p$ collisions at $\sqrt{s}=63 \mathrm{GeV}$, as measured by the SFM collaboration [3] at the ISR. These data show a low- $p_{T}$ enhancement reminiscent of the SPS $A B$ results in a truly baryon-depleted central region, so that contributions from $\Delta \rightarrow N \pi$ are reduced [4]. Moreover, these experiments are closer to RHIC energies!

\section{PARTIAL THERMALIZATION}

In high energy $p p$ reactions as in nuclear collisions at the SPS, the dynamics of the central region is dominated by pions at proper times $>t_{0} \sim 1-3 \mathrm{fn}_{\mathrm{i}}$ when hadronic degrees of freedom become well defined. Longitudinal expansion rarefies the system and inhibits thermalization during this 'late' period, because the pion scattering rate decreases strongly as the density drops. The pion density at midrapidity $n \propto t^{-1}$ falls as the proper time $t$ increases. This dilution reduces the effective scattering rate $\nu \approx\langle\sigma v\rangle n$ roughly as $t^{-1}$, since the density dependence of the momentum transfer cross section $\langle\sigma v\rangle$ is weak $[5,6]$. Significantly, the expansion rate of the system $|\dot{n}| / n$ also falls as $t^{-1}$.

In [1] I suggested that the rapid longitudinal expansion prevents the pion fluid from reaching local equilibrium but, rather, drives the system towards a novel partially thermalized state. In essence, the macroscopic evolution is never strictly 'quasi-static' on microscopic scales. To exhibit the character of partially thermalized flow, I determined the energy density $\mathcal{E}$ on the central slice as a function of time $t$ using the Boltzmann equation with the density dependent relaxation rate $\nu \propto n$. I find

$$
\mathcal{E}(t) \approx \mathcal{C}\left(t_{0} / t\right)^{1+\gamma}
$$




\section{DISCLAIMER}

This report was prepared as an account of work sponsored by an agency of the United States Government. Neither the United States Government nor any agency thereof, aor any of their employees, makes any warranty, express or implied, or assumes any legal liability or responsibility for the accuracy, completeness, or usefulness of any information, apparatus, product, or process disclosed, or represents that its use would not infringe privately owned rights. Reference herein to any specific commercial product, process, or service by trade name, trademark, manufacturer, or otherwise does not necessarily constitute or imply its endorsement, recom. mendation, or favoring by the United States Government or any agency thereof. The views and opinions of authors expressed herein do not necessarily state or reflect those of the United States Government or any agency thereof. 

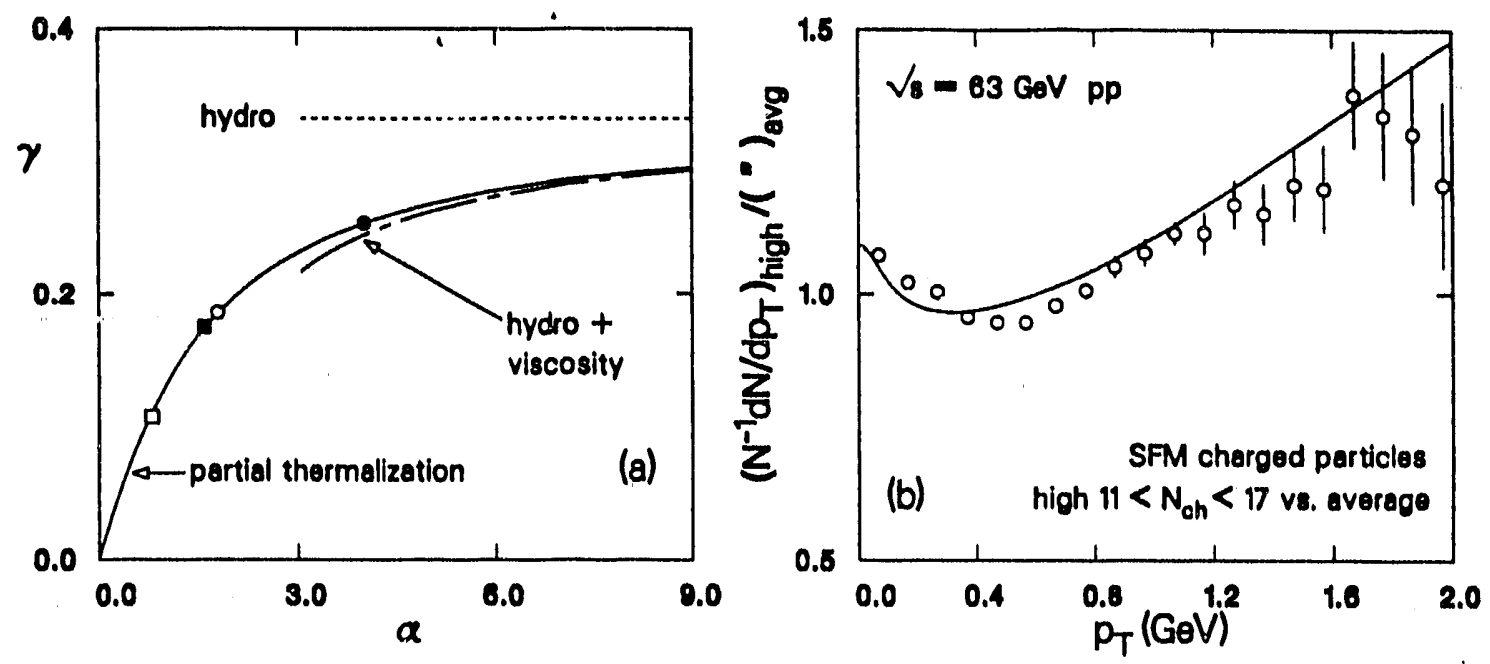

Figure 1: (a) Degree of thermalization $\gamma$ as a function of the coupling parameter $\alpha$. Expected $\gamma$ for ISR high multiplicity and minimum bias $p p$ (filed and empty squares), and SPS $\mathrm{S}+\mathrm{S}$ (empty circles) and $\mathrm{O}+\mathrm{Au}$ (filled circles). (b) ISR $p p$ data [3] compared to calculated ratio of $d N / d p_{T}$.

where the coefficient $\mathcal{C}$ depends on the initial conditions. The exponent $\gamma$ in fig. 1a varies continuously between the noninteracting and ideal hydrodynamic extremes, 0 and $1 / 3$, depending only on the coupling parameter:

$$
\alpha \equiv \nu n /|\dot{n}|=\langle\sigma v\rangle\left(\pi R^{2}\right)^{-1} d N / d y
$$

where $R$ is the projectile radius and $d N / d y$ is the pion rapidity density [1]. I extract $\langle\sigma v\rangle \approx 7-10 \mathrm{mb}$ from variational [5] and Chapman-Enskog [6] calculations based on $\pi \pi$ scattering data. Although (1) strictly applies for $t \gg t_{0}$, convergence is rapid for physically plausible initial conditions.

The exponent $\gamma$ characterizes the degree of thermalization of the expanding fluid, as seen by comparing $\gamma(\alpha)$ to the hydrodynamic limit in fig. 1a. Hydrodynamics including viscosity describes the flow at the $20 \%$ level for $\alpha>4$, while ideal, viscosity free hydrodynamics is only valid as $\alpha \rightarrow \infty$. This measure of thermalization is equivalent to the more familiar flow anisotropy [7], since $\gamma=P_{\|} /\left(2 P_{\perp}+P_{\|}\right)$where $P_{\|}$and $P_{\perp}$ are the longitudinal and transverse pressures at midrapidity in configuration space [1]. The partially thermalized state corresponds to a fixed, steady anisotropy $P_{\|}>P_{\perp}$.

To estimate the degree of thermalization expected in nuclear collisions for the central region at the SPS, observe that NA35 reports $d N^{-} / d y \approx 27$ and 41 for charged particles from $\mathrm{S}+\mathrm{S}$ and $\mathrm{O}+\mathrm{Au}$. The $\gamma(\alpha)$ expected for $\mathrm{O}+\mathrm{Au}$ and $\mathrm{S}+\mathrm{S}$ are indicated in fig. 1a by the filled and empty circles, respectively. The fluid in $\mathrm{O}+\mathrm{Au}$ is marginally in the viscous flow regime with $\alpha \approx 4$, while $\mathrm{S}+\mathrm{S}$ is in an intermediate regime with $\alpha \approx 1.8$.

\section{PION CONSERVATION}

The pion number is approximately conserved in the late stages of $A B$ or $p p$ collisions, because inelastic pion interactions are too slow to vary the number of pions in the short lived hadronic fluid $[8,5,9]$. In a static gas, reactions such as $\pi \pi \rightleftharpoons K \bar{K}$ and $\pi \pi \rightleftharpoons \rho \rho$ maintain chemical equilibrium, so that the pion chemical potential $\mu_{\pi}$ vanishes. 

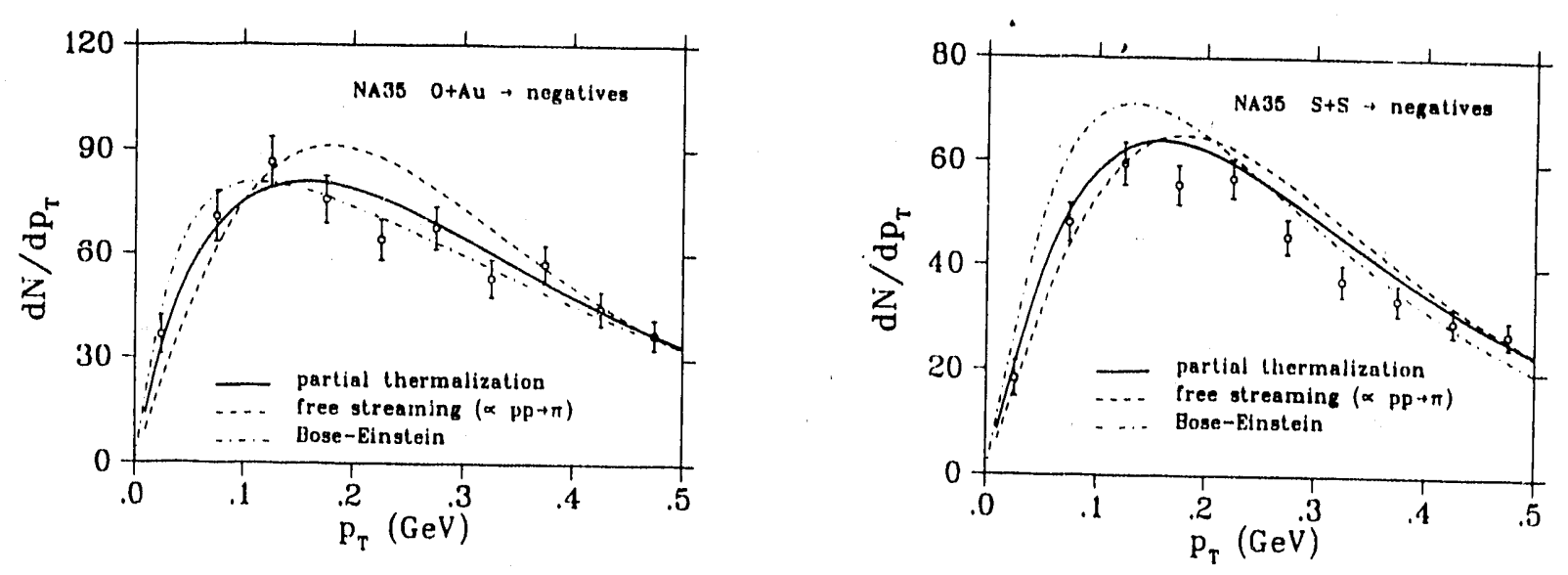

Figure 2: Transverse momentum distributions for $\mathrm{O}+\mathrm{Au}$ and $\mathrm{S}+\mathrm{S}$ [2].

However, these processes do not affect the pion distribution appreciably during the $<5-$ $10 \mathrm{fm}$ duration of a nuclear collision, as rate estimates variously based on $\pi \pi$ scattering data and chiral perturbation theory indicate. Baym realized that the expansion of the collision volume can then generate a nonzero $\mu_{\pi}[8]$.

Kataja and Ruuskanen recently observed that the pion fluid can be initially superdense, i.e. its density at $t_{0}$ can exceed the chemical equilibrium value corresponding to the initial $\left\langle p_{T}\right\rangle[10]$. Indeed, the pion fluid in $\mathrm{O}+\mathrm{Au}$ would have a large initial chemical potential, roughly $3 m_{\pi} / 4$, if the fluid were in thermal equilibrium: To estimate the pion density [2], we take the $d N / d y \approx 3 \pi R_{A}{ }^{2} n\left(t_{0}\right) t_{0} / 2$ applicable at midrapidity where the factor $\approx 3 / 2$ accounts for resonance decays. NA35 O+Au data then imply $n\left(t_{0}\right) \approx 1 \mathrm{fm}^{-3}$ at $t_{0} \sim 3 \mathrm{fm}$. This estimate exceeds the $\mu_{\pi}=0$ value $n_{\text {eq }} \approx 0.15 \mathrm{fm}^{-3}$ at the effective temperature $\approx\left\langle p_{T}\right\rangle / 2 \approx 160 \mathrm{MeV}$ that describes the $p_{T}$ spectrum (we assume $\left\langle p_{T}\right\rangle \approx\left\langle p_{T}\right\rangle_{N N}$ for pions formed in $N N$ subcollisions).

In a superdense pion fluid, Ruuskanen and I find that the number-conserving $\pi \pi$ interactions shift the convex shape of the initial spectrum at low $p_{T}$ in agreement with the concave form of the NA35 O+Au data (the "low $p_{T}$ enhancement" discussed in refs. cited in [2] and Sarabura's talk in these proceedings). To obtain the pion spectra in fig. 2, we again solve the Boltzmann equation for conserved, Bose pions using the relaxation rate from [1]. We assume that pions are formed in independent $N N$ subcollisions to fix the initial spectrum (see [2] for details). The calculated low $p_{T}$ enhancement reflects the low momentum peaking of the thermal equilibrium Bose-Einstein distribution for $\mu_{\pi}>0$ relative to the chemical equilibrium distribution $[9,10,11]$. Because the system is not fully thermalized, however, the final state cannot be represented by a single $T$ and $\mu_{\pi}$ at the instant of freezeout.

Recently, Welke and Bertsch have performed Boltzmann equation calculations with an exact numerical treatment of the $\pi \pi$ collision term [12]. They obtain both $p_{T}$ and $y$ distributions for $\mathrm{O}+\mathrm{Au}$, and find qualitative agreement with our results at midrapidity.

I emphasize that both the superdense initial conditions and the partial thermalization of the flow are needed to correctly interpret the qualitative low momentum behavior. The hydrodynamic evolution of $\mu_{\pi}$ is sensitive to the entropy per particle $s / n$ through the Gibbs-Duhem relation $\mu_{\pi} / T=(\mathcal{E}+P) / n T-s / n$. Adiabatic expansion 
( $s / n=$ const.) drives $\mu_{\pi}$ to positive values even if one takes $\mu_{\pi}\left(t_{0}\right)=0[8,9]$. However, entropy is gener ated by deviations from local thermal equilibrium. The estimated entropy increase from shear viscosity corrections in hydrodynamics [5] can drive $\mu_{\pi}$ to negative values for $\mu_{\pi}\left(t_{0}\right)=0$. Therefore the large initial densities [10] are necessary, provided that one accepts the estimates of viscous corrections $[5,6]$ based on the free space $\pi \pi$ cross sections. Of course, the in-medium effects discussed by Shuryak in these proceedings may change the entire picture [13] - many questions remain!

\section{TOWARDS EQUILIBRIUM}

Following [2], I have calculated the $p_{T}$ spectra for charged particles in high multiplicity and minimum bias $p p$ collisions at $\sqrt{s}=63 \mathrm{GeV}$ for comparison to SFM measurements [3]. The filled and empty squares in fig. 1a indicate the estimated degree of thermalization for high multiplicity and average $p p$ collisions at $\sqrt{s}=63 \mathrm{GeV}$. Like $\mathrm{S}+\mathrm{S}$, the pion fluid in the high multiplicity $p p$ reactions fall midway between the free streaming and hydrodynamic regimes. In applying (2) to $p p \rightarrow \pi X$, I take $\pi R^{2} \approx \sigma_{\mathrm{pp}}$ and $(d N / d y)_{\text {high }} \approx 2(d N / d y)_{\text {avg }} \approx 8.5$ extrapolated from the SFM charged particle multiplicities and $d N / d y$. The spectrum at the $p p$ initial time $t_{0} \approx 1 \mathrm{fm}$ is taken so that $\left\langle p_{T}\right\rangle \approx 0.38 \mathrm{GeV}$ at the higher energy [3]; otherwise the initial distribution is the same as for SPS energies [2]. The results in fig. $1 \mathrm{~b}$ are in good agreement with the data.

Figure 1a implies an increase in the degree of thermalization from $p p$ to $\mathrm{S}+\mathrm{S}$ to $\mathrm{O}+\mathrm{Au}$ following the increase in $\alpha \propto A^{-2 / 3} d N / d y$. This trend is consistent with the behavior of the calculated and measured spectra in figs. $1 \mathrm{~b}$ and 2. In particular, the partially thermalized distribution for $\mathrm{O}+\mathrm{Au}$ is practically indistinguishable from the fully thermalized Bose distribution. The $\mathrm{O}+\mathrm{Au}$ system is not fully thermalized, however, so that the link between the equilibrium equation of state final state quantities, e.g. $\left\langle p_{T}\right\rangle$ and $d N / d y$, is extremely subtle. Nevertheless, the trend that heavier systems are more thermalized is encouraging.

I am grateful to G. Baym and V. Ruuskanen for their enjoyable coliaboration, and to M. Prakash, M. Tannenbaum, R. Venugopalan, and G. Welke for stimulating discussions.

\section{REFERENCES}

1 S. Gavin, Nucl. Phys. B351 (1991) 561.

2 S. Gavin and P. V. Ruuskanen, Phys. Lett. B262 (1991) 326.

3 W. Bell et al. Z. Phys. C27 (1085) 191.

4 J. Sollfrank, P. Koch, and U. Heinz, TPR-91-17, and refs. therein.

5 S. Gavin, Nucl. Phys. A435 (1985) 561.

6 M. Prakash, M. Prakash, R. Venugopalan and G. Welke, SUNY-NTG-91-2 (1991).

7 P. Danielowicz and G. Odyniec, Phys. Lett. 175B (1985) 146.

8 G. Baym, Quark Marter '84, Lect. Notes in Phys. 221 (Springer, 1984) p. 39.

9 P. Gerber et al. Phys. Lett. B246 (1990) 513.

10 M. Kataja and P. V. Ruuskanen, Phys. Lett. B243 (1990) 181.

11 J. Zimányi, G. Fai, and B. Jacobsson, Phys. Rev. Lett. 43 (1979) 1705.

12 G.M. Welke and G.F. Bertsch, MSUCL-772 (1991).

13 A. Schenk, Nucl. Phys. B363 (1991) 97. 

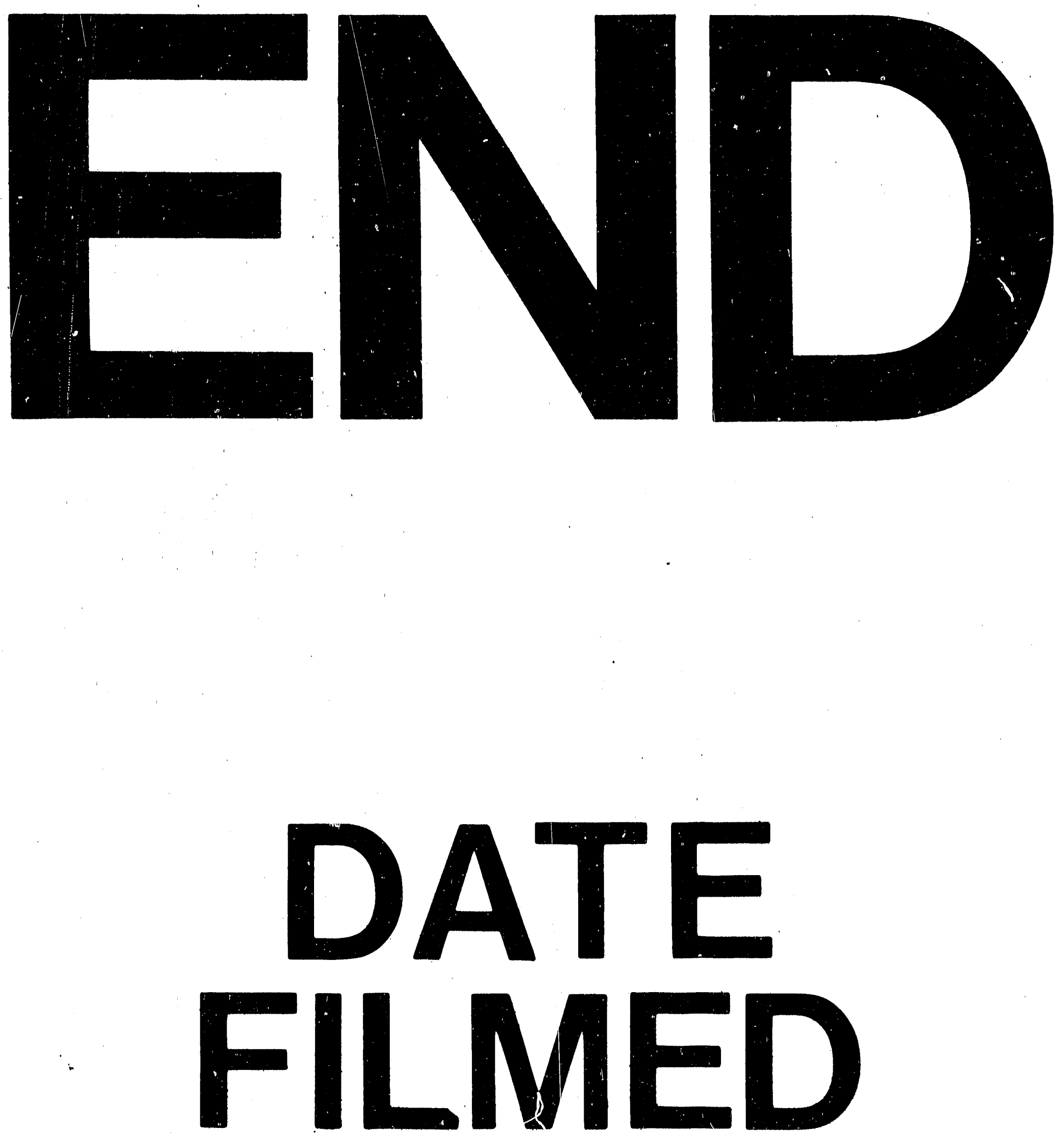

1

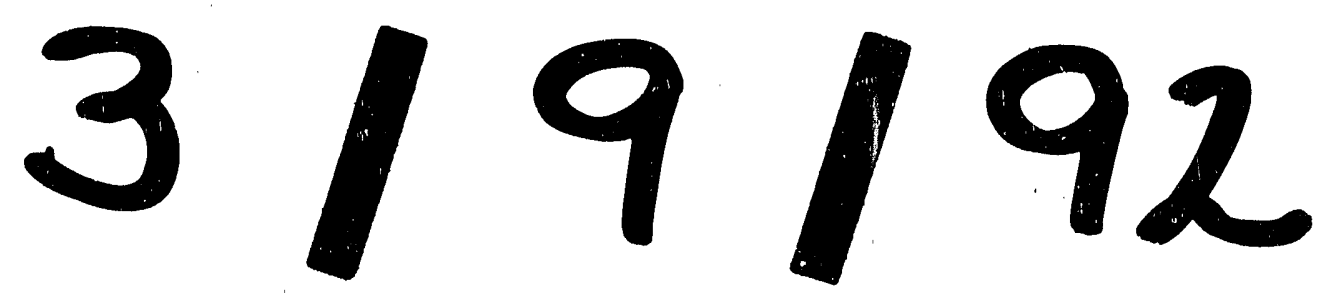


